\title{
Photosystem II core quenching in desiccated Leptolyngbya ohadii
}

\author{
Reza Ranjbar Choubeh ${ }^{1} \cdot$ Leeat Bar-Eyal ${ }^{2} \cdot$ Yossi Paltiel $^{3} \cdot$ Nir Keren $^{2} \mathbb{D} \cdot$ Paul C. Struik $^{4} \cdot$ Herbert van Amerongen $^{1,5}$
}

Received: 6 May 2019 / Accepted: 10 September 2019 / Published online: 18 September 2019

(c) The Author(s) 2019

\begin{abstract}
Cyanobacteria living in the harsh environment of the desert have to protect themselves against high light intensity and prevent photodamage. These cyanobacteria are in a desiccated state during the largest part of the day when both temperature and light intensity are high. In the desiccated state, their photosynthetic activity is stopped, whereas upon rehydration the ability to perform photosynthesis is regained. Earlier reports indicate that light-induced excitations in Leptolyngbya ohadii are heavily quenched in the desiccated state, because of a loss of structural order of the light-harvesting phycobilisome structures (Bar Eyal et al. in Proc Natl Acad Sci 114:9481, 2017) and via the stably oxidized primary electron donor in photosystem I, namely P700+ (Bar Eyal et al. in Biochim Biophys Acta Bioenergy 1847:1267-1273, 2015). In this study, we use picosecond fluorescence experiments to demonstrate that a third protection mechanism exists, in which the core of photosystem II is quenched independently.
\end{abstract}

Keywords Photosystem II quenching · Time-resolved fluorescence spectroscopy $\cdot$ Cyanobacteria $\cdot$ Photoprotection

\section{Introduction}

Cyanobacteria are photosynthetic organisms which are abundant in aquatic and terrestrial environments. Terrestrial cyanobacteria constitute $28 \%$ of total global cyanobacterial biomass and $79 \%$ of this is constituted by cyanobacteria living in arid soil crusts (Garcia-Pichel et al. 2003).

Filamentous cyanobacteria live in biological desert soil crusts and face harsh environmental conditions such as high

Electronic supplementary material The online version of this article (https://doi.org/10.1007/s11120-019-00675-0) contains supplementary material, which is available to authorized users.

Herbert van Amerongen

herbert.vanamerongen@wur.nl

1 Laboratory of Biophysics, Wageningen University, Wageningen, The Netherlands

2 Department of Plant \& Environmental Sciences, The Alexander Silberman Institute of Life Sciences, The Hebrew University of Jerusalem, Jerusalem, Israel

3 Applied Physics Department, The Hebrew University of Jerusalem, Jerusalem, Israel

4 Centre for Crop Systems Analysis, Wageningen University, Wageningen, The Netherlands

5 MicroSpectroscopy Research Facility, Wageningen University, Wageningen, The Netherlands sun light intensity, hydration/rehydration cycles, high daytime, and low nighttime temperatures. These cyanobacteria become desiccated in the hot sunlight and stop their photosynthetic activity. However, they are well adapted to cope with this desiccation and in low light they regain their photosynthetic activity after rehydration (Raanan et al. 2016a, b). High light intensity also challenges these cyanobacteria creating a need for quenching mechanisms to protect the photosynthetic apparatus. Without such protection, reactive oxygen can be produced, which is harmful for the cell. Cyanobacteria have three main photosynthetic antenna systems: phycobilisomes (PBSs), the core of photosystem II (PSII), and the core of photosystem I (PSI). Previous studies have shown that PBSs in the desiccated state lose their organized structure leading to quenching of excitation energy and the loss of efficient energy transfer capacity. Upon rehydration this capacity is regained (Bar Eyal et al. 2015, 2017; Eisenberg et al. 2017). In addition, oxidized PSI was suggested as a quencher of excitation energy in the desiccated state (Bar Eyal et al. 2015).

In this work, we study Leptolyngbya ohadii, grown at low light intensities $\left(60 \mu \mathrm{mol}\right.$ photons $\left.\mathrm{m}^{-2} \mathrm{~s}^{-1}\right)$, by timeresolved fluorescence spectroscopy. We provide evidence that besides quenching of the light-harvesting PBS and PSI, also direct PSII excitation energy quenching occurs in the desiccated state. 


\section{Materials and methods}

\section{Culture growth and sample preparation}

The Leptolyngbya ohadii cells were grown and prepared in their hydrated and desiccated state as reported in Bar Eyal et al. $(2015,2017)$. Cultures were incubated in YBG11 medium in shaking flasks (Harel et al. 2004), at $30{ }^{\circ} \mathrm{C}$ and $60 \mu \mathrm{mol}$ photons $\mathrm{m}^{-2} \mathrm{~s}^{-1}$. Cells were homogenized and placed on nitrocellulose filters. The filters were air dried at room temperature for 1 day prior to spectroscopic measurements. The minimal amount of time required for reaching the stable desiccated state was $2 \mathrm{~h}$ (Bar Eyal et al. 2017).

\section{Time-resolved measurements and data analysis}

Time-resolved fluorescence of the cells was recorded at room temperature using a picosecond streak-camera system (van Stokkum et al. 2008) as reported before (Bar Eyal et al. 2017; Ranjbar Choubeh et al. 2018), using a time window of 800 ps. The frequency-doubled output of a Ti:sapphire laser (Coherent, Mira) (800 nm) was used to excite the samples at $400 \mathrm{~nm}$. Excitation power was $\sim 100 \mathrm{nW}$ (repetition rate $4 \mathrm{MHz}$ ) and the spot size was $\sim 0.1 \mathrm{~mm}$. The full-width at half-maximum of the instrument response function (IRF) was $\sim 8$ ps. Data were globally analysed with the use of Glotaran (Snellenburg et al. 2012) and the TIMP package (Mullen and van Stokkum 2007) for the statistical computing and graphics software R. The data from four experiments (four experiments in hydrated state and four experiments in desiccated state) was fitted to a sum of exponential functions convoluted with the IRF (Lakowicz 2006) and the relatively broad IRF determines the shortest time profile that our streak-camera system can measure. A narrow full-widthhalf-maximum for the Gaussian function correlates with a better time resolution. For our instrument, we could model the IRF as a Gaussian function. The measured fluorescence is obtained as

$\mathrm{F}(t, \lambda)=\int_{0}^{t} \operatorname{IRF}(t-x, \lambda) \times \sum_{i} \operatorname{DAS}_{i}(\lambda) \times \exp \left(k_{i} x\right) \mathrm{d} x$,

where $\mathrm{F}(t, \lambda)$ is the fluorescence, $\mathrm{t}$ is the time after excitation, $\lambda$ is the wavelength, $k_{i}$ is the rate by which the $i$ th exponential decays, and $\operatorname{DAS}_{i}(\lambda)$ (decay-associated-spectrum) are the amplitudes at the different wavelengths of the $i$ th exponential function. $x$ indicates time and the integral is performed up to time $t$. By fitting the recorded fluorescence to $\mathrm{F}(t, \lambda)$ the rate constants $k_{i}$ and the $\mathrm{DAS}_{i}$ are determined. For examples of fit quality, see Figs. S1-S5.

\section{Results}

Figure 1 shows streak-camera images for the hydrated and desiccated states for which an excitation wavelength of $400 \mathrm{~nm}$ was used. Light of this wavelength preferentially excites chlorophylls but some phycobilisome excitation is unavoidable. The four red vertical lines mark the wavelengths $640 \mathrm{~nm}$ (C-phycocyanin rods (CPC rods)), $660 \mathrm{~nm}$ (allophycocyanin (APC) 660), $690 \mathrm{~nm}$ (mainly Chlorophyll (Chl) $a$ of PSII and some APC680), and $715 \mathrm{~nm}$ (Chl $a$ of PSI). In the desiccated state, the lifetime of the excited-state population is substantially shorter, especially at $690 \mathrm{~nm}$, which reflects the quenching of excitation energy. The time traces at these wavelengths are shown in Fig. 2.

Figure $3 \mathrm{a}-\mathrm{d}$ shows the result of a global analysis of timeresolved fluorescence of cells in their desiccated and in their hydrated states. Using the singular value decomposition method (van Stokkum et al. 2004), it was concluded that four lifetimes are required to fit the data for both states. No meaningful fitting was possible with 5 lifetimes. The time-zero spectrum presents the fluorescence emission spectrum directly after excitation assuming that no other relaxation processes occur, and it was calculated by summing all the DAS. This spectrum is shown in Fig. 3e. All the DAS
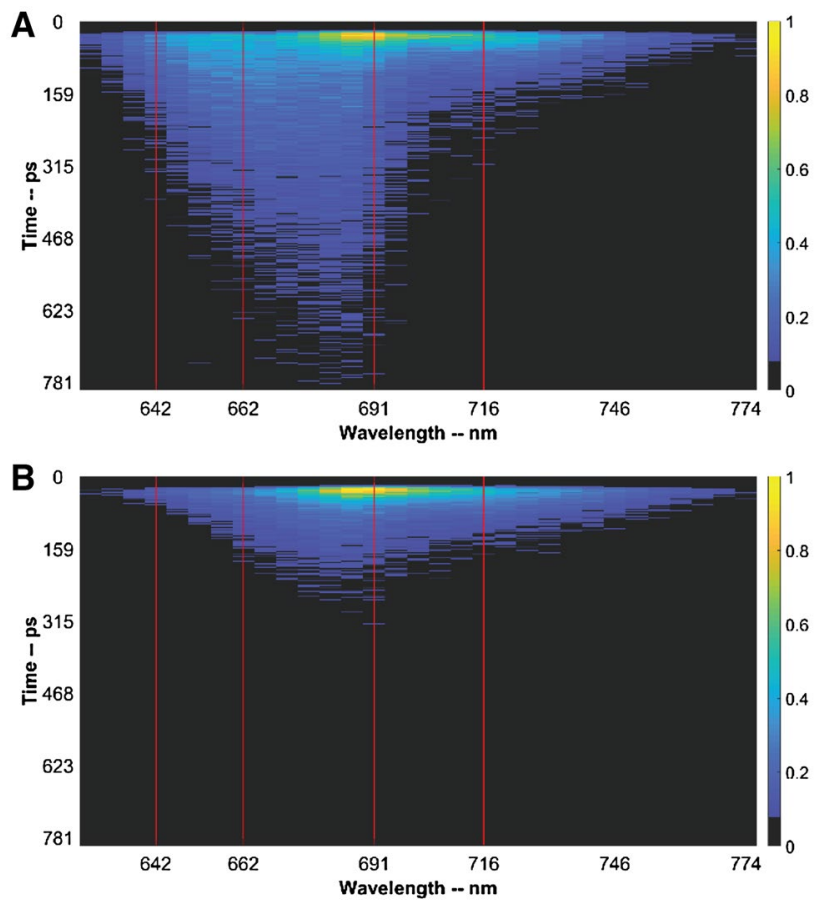

Fig. 1 Intensity colour-coded streak-camera images of $L$. ohadii in hydrated (a) and desiccated (b) state at room temperature. The lifetime of the excited state shortens in the desiccated state. The time traces along the red vertical lines are shown in Fig. 2. The excitation wavelength is $400 \mathrm{~nm}$ (mainly exciting Chl $a$ ) 

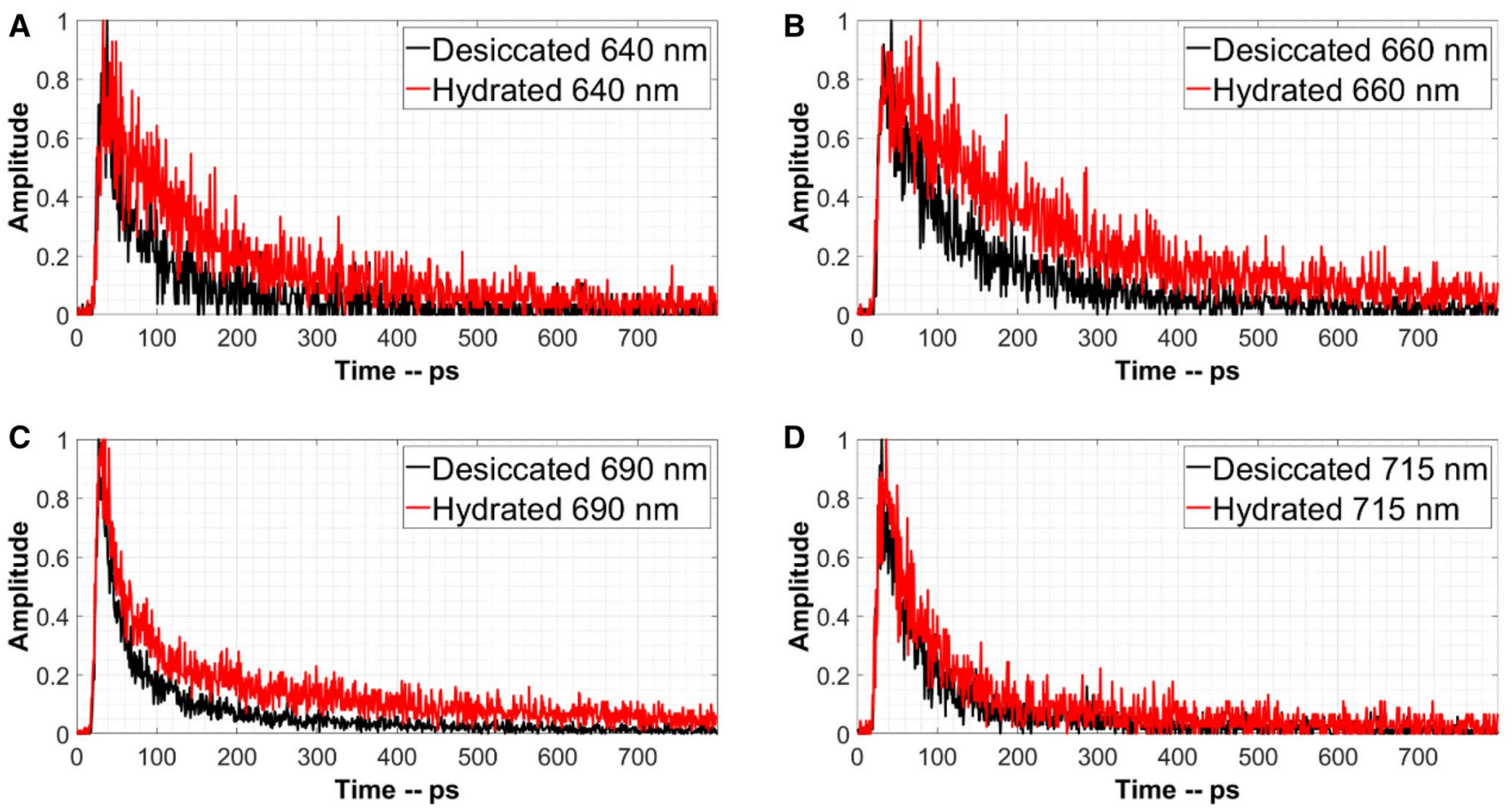

Fig. 2 Time traces at the wavelengths marked in Fig. 1 by vertical red lines. In the desiccated state the excited-state lifetime shortens, especially at $690 \mathrm{~nm}$. The excitation wavelength is $400 \mathrm{~nm}$

corresponding to the hydrated state are presented in Fig. $3 \mathrm{f}$ showing the relative amplitude of different DAS. The fastest component that could be resolved with global analysis has a 10-ps lifetime, both for hydrated and desiccated cells. The corresponding decay-associated spectra (DAS) for both states are given in Fig. 3a. This DAS reflects excitation energy transfer (EET) within the CPC rods of the phycobilisomes (Tian et al. 2011, 2012), manifested by the negative band between 627 and $660 \mathrm{~nm}$. EET within PSI towards long-wavelength $\mathrm{Chl} a$ is reflected by the combination of a positive band around 690 and a negative one near $720 \mathrm{~nm}$ (Tian et al. 2013).

Figure $3 \mathrm{~b}$ shows 3 positive peaks for both hydrated and desiccated states at $640 \mathrm{~nm}$ (CPC rods), $690 \mathrm{~nm}$ (mainly Chl $a$ and some APC680), and $710 \mathrm{~nm}$ (PSI). In the hydrated state, the negative peak at $660 \mathrm{~nm}$ corresponds to APC660 and it indicates EET transfer from CPC rods to APC660. A previous study (Bar Eyal et al. 2017) showed that the excitation energy is quenched within CPC rods in the desiccated state, which is reflected by the positive peak at $660 \mathrm{~nm}$ in Fig. 3b. Note that in the hydrated state in which EET is not quenched the amplitude in Fig. $3 \mathrm{~b}$ at $660 \mathrm{~nm}$ is negative which indicates EET. The lack of EET to APC660 in the desiccated state shows up as a lack of negative amplitude around $660 \mathrm{~nm}$. The amplitude of the DAS in the PSI emission region $(>700 \mathrm{~nm})$ is the same for the desiccated and the hydrated state; however, the fluorescence lifetime is shorter in the desiccated state (22 ps) than in the hydrated state (33 ps), which demonstrates that also PSI is being quenched, in agreement with the data presented before (Bar Eyal et al. 2015).

Figure $3 \mathrm{c}$ shows the next DAS for the desiccated and the hydrated states, both corresponding to a 98-ps lifetime. The APC660 peak at $660 \mathrm{~nm}$, which is prominently present in the hydrated state, is substantially reduced in the desiccated state. This is due to the lack of EET from CPC to APC660 in the desiccated state as was already clear from Fig. $3 \mathrm{~b}$. The peaks at $690 \mathrm{~nm}$ and $720 \mathrm{~nm}$ belong to PSII and PSI, respectively, which have an increased amplitude in the desiccated state. The increase in amplitude is accompanied by a concomitant decrease in amplitude in the same wavelength region of the long-lifetime component, as shown in Fig. 3d. This increase in amplitude of the short-lived component at the expense of the amplitude of the long-lived component largely reflects the fluorescence quenching. This phenomenon can also be observed in the calculated steady-state spectrum shown in Fig. 4 as a reduction in the fluorescence amplitude.

Figure $3 \mathrm{~d}$ shows 3 bands around $660 \mathrm{~nm}$ (APC660), $690 \mathrm{~nm}$ (mainly Chl $a$ of PSII and some APC680), and 720 nm (PSI). The peak at $660 \mathrm{~nm}$ is substantially reduced in the desiccated state, because the excitation energy does not reach APC660 and is quenched in the CPC rods as reported previously (Bar Eyal et al. 2017). Also, the peaks of PSII and PSI at $690 \mathrm{~nm}$ 

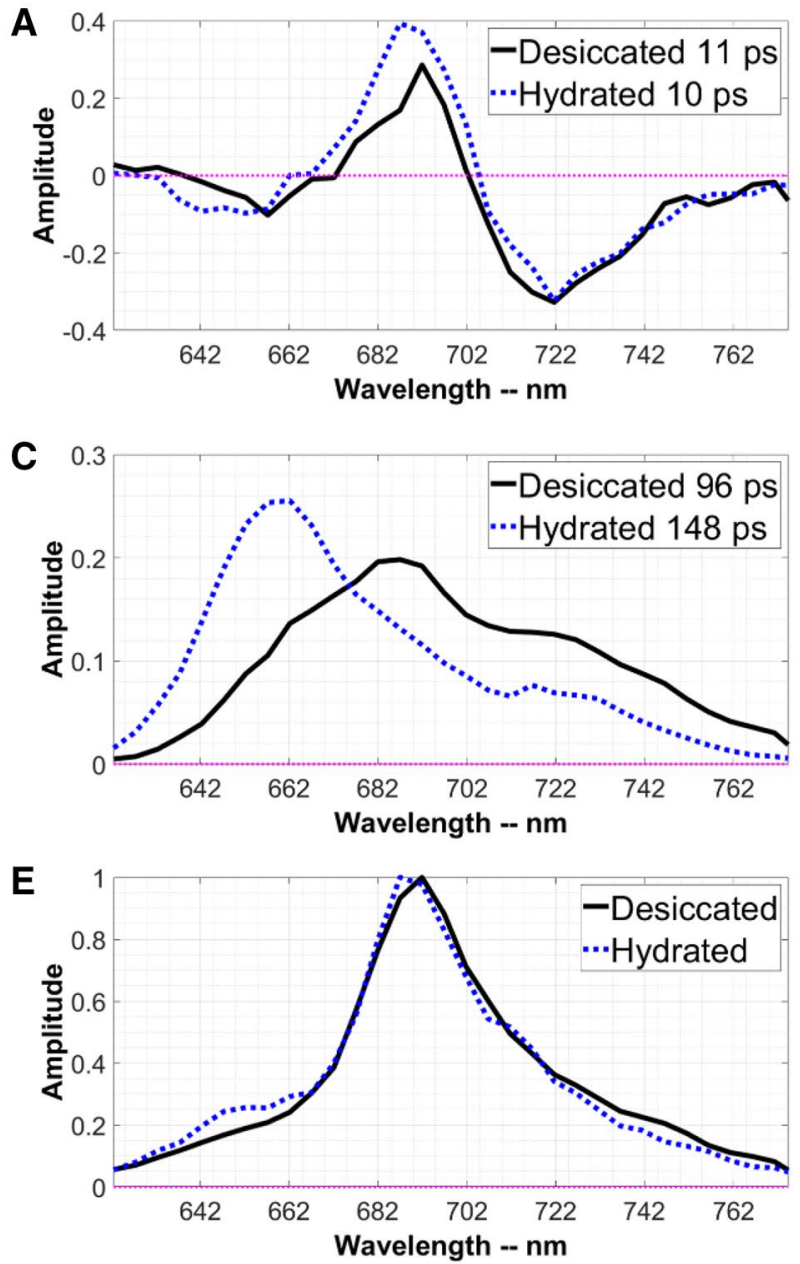

Fig. 3 Global analysis of time-resolved fluorescence data of the cyanobacterium $L$. ohadii excited at $400 \mathrm{~nm}$ in its desiccated and hydrated state. a-d The DAS and corresponding lifetimes obtained using global analysis. e The time-zero spectrum, which is the fluo-

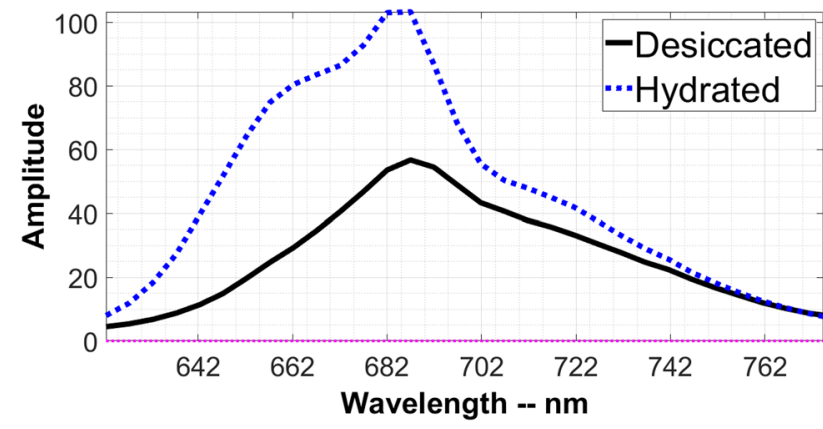

Fig. 4 The calculated steady-state spectra of $L$. ohadii in both the desiccated and hydrated state. The fluorescence is substantially quenched in the desiccated state at 640-660 nm (CPC rods and APC660), $690 \mathrm{~nm}$ (mainly Chl $a$ of PSII and some APC680), and to a lesser extent at $715 \mathrm{~nm}$ (Chl $a$ of PSI)
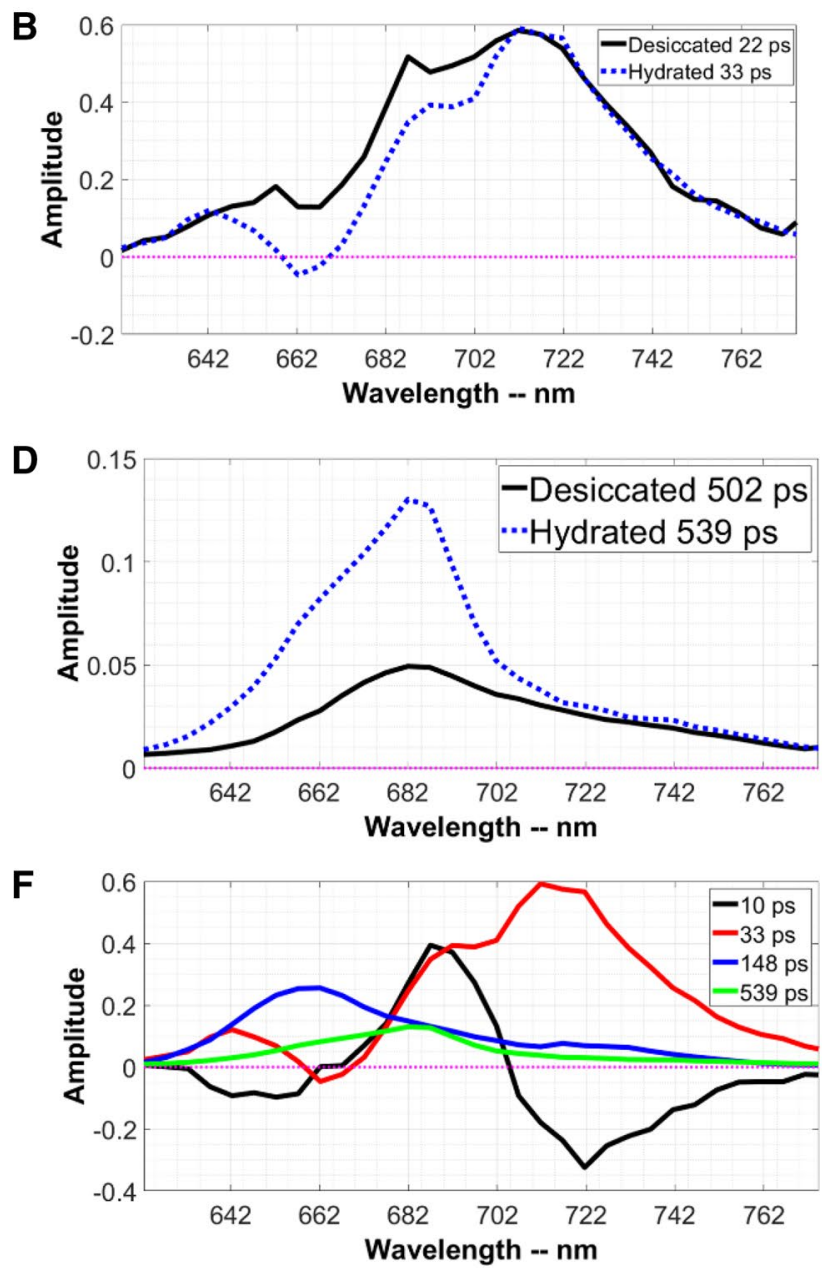

rescence emission just after excitation and after all ultrafast relaxation processes are finished. The time-zero spectrum was calculated by summing all the DAS in a-d for each state. f All the DAS of L. ohadii in the hydrated state to show their relative amplitudes

and $720 \mathrm{~nm}$ are reduced in the desiccated state. This reduction is accompanied by an increase of the amplitude in the DAS of Fig. $3 \mathrm{~b}$ and c. Both results indicate that PSI and PSII are also directly quenched in the desiccated state. The change in the amplitude of the PSII emission is greater than that of PSI, which shows PSII is quenched to a greater extent than PSI.

These experiments were also performed in the presence of a saturating concentration of DCMU, a PSII inhibitor that binds to the $Q_{B}$ site and inhibits photochemical energy transfer (see Fig. S6). The differences observed between hydrated and desiccated states were similar, demonstrating that the effect is not photochemical in nature. 


\section{Discussion}

Desert-dwelling cyanobacteria need to protect themselves against high light intensity during the day, which in their natural habitat is accompanied by drought. It was reported before that these cyanobacteria do this by quenching the excitation energy within the CPC rods of the PBSs (Bar Eyal et al. 2017) and PSI Chl $a$ molecules (Bar Eyal et al. 2015). The results in the present study confirm those findings but, in addition, suggest that the core of PSII is also quenched in the desiccated state. This finding matches a pattern that is observed in different types of organisms. It was observed before that in diatoms part of the antenna system from the PSII core uncouples in high light conditions, while both the uncoupled antenna and the PSII core become quenched separately (Miloslavina et al. 2009; Chukhutsina et al. 2014). A similar pattern has been proposed for photoprotective non-photochemical quenching (NPQ) in plants by Holzwarth and coworkers [for an overview see (Jahns and Holzwarth 2012)] in which fast quenching is ascribed to the detachment of lightharvesting complexes accompanied by their aggregation and quenching, followed by quenching of the PSII core plus attached antenna complexes on a slower time scale. Both for diatoms and plants, this quenching of the core plus attached antenna complexes is thought to relate to the involvement of carotenoids via a xanthophyll cycle, but such a cycle is absent in cyanobacteria.

Among the desiccating organisms, lichens seem to make use of two quenching mechanisms, one is operating at the level of the antenna and the other, which was attributed to spill-over from PSII to PSI, is operating at the level of the PSII core (Slavov et al. 2013). In principle, spill-over might contribute to the quenching of PSII in L. ohadii. This might be detected via the disappearance of PSII emission and the concomitant appearance of PSI emission. However, we do not find any convincing proof for that. The increase of the fluorescence above $700 \mathrm{~nm}$ in Fig. $3 b$ in the desiccated state is due to a rise in PSII emission, whereas the peak of PSI at $720 \mathrm{~nm}$ is more or less the same for the desiccated and hydrated states. This is reminiscent of the situation in the cyanobacterium Synechococcus elongatus 7942 in which quenching of the PSII core was observed upon going from state I to state II. Also in that case spill-over could be ruled out because no such change in PSI emission was observed (Ranjbar Choubeh et al. 2018). The most likely explanation for quenching of the PSII core is non-radiative energy dissipation within its reaction centre. This has been suggested as a highly effective protective mechanism against photodamage upon excessive excitation in cyanobacteria before (Sane et al. 2002; Cser and Vass 2007). Furthermore, the possibility of non-radiative excitation energy quenching occurring within the reaction centres of PSII and its functional/ physiological implications have been discussed for various photosynthetic organisms (Ivanov et al. 2003, 2008). Similar conclusions have been reported in a number of studies on desiccated lichens, and they all emphasize the role of an auxiliary quenching mechanism within the PSII reaction centre (Heber 2008; Heber et al. 2006a, b, 2007, 2011).

In conclusion, like many other photosynthetic organisms, also $L$. ohadii makes use of multiple photoprotective quenching mechanisms. In this particular organism, one leads to the quenching of the antenna and two others cause the quenching of the cores of PSI and PSII, respectively. However, the underlying physical model for the novel mechanism described here remains to be elucidated.

Acknowledgements This work was funded by the Netherlands Organization for Scientific Research (NWO) (Project Number 10TBSC24-3) and the Israeli Science Foundation Grant NSFC-ISF 2466/18 awarded to NK and YP. This project was carried out within the research programme of BioSolar Cells, co-financed by the Dutch Ministry of Economic Affairs, through grant FOM24. We thank Dr Arjen Bader for his technical support during the time-resolved measurements.

\section{Compliance with ethical standards}

Conflict of interest The authors declare that they have no conflict of interest.

Open Access This article is distributed under the terms of the Creative Commons Attribution 4.0 International License (http://creativeco mmons.org/licenses/by/4.0/), which permits unrestricted use, distribution, and reproduction in any medium, provided you give appropriate credit to the original author(s) and the source, provide a link to the Creative Commons license, and indicate if changes were made.

\section{References}

Bar Eyal L, Eisenberg I, Faust A, Raanan H, Nevo R, Rappaport F, Krieger-Liszkay A, Sétif P, Thurotte A, Reich Z, Kaplan A, Ohad I, Paltiel Y, Keren N (2015) An easily reversible structural change underlies mechanisms enabling desert crust cyanobacteria to survive desiccation. Biochim Biophys Acta Bioenergy 1847:12671273. https://doi.org/10.1016/j.bbabio.2015.07.008

Bar Eyal L, Ranjbar Choubeh R, Cohen E, Eisenberg I, Tamburu C, Dorogi M, Ünnep R, Appavou M-S, Nevo R, Raviv U, Reich Z, Garab G, van Amerongen H, Paltiel Y, Keren N (2017) Changes in aggregation states of light-harvesting complexes as a mechanism for modulating energy transfer in desert crust cyanobacteria. Proc Natl Acad Sci 114:9481. https://doi.org/10.1073/pnas.17082 06114

Chukhutsina VU, Büchel C, van Amerongen H (2014) Disentangling two non-photochemical quenching processes in Cyclotella meneghiniana by spectrally-resolved picosecond fluorescence at 77 K. Biochim Biophys Acta Bioenergy 1837:899-907. https:// doi.org/10.1016/j.bbabio.2014.02.021 
Cser K, Vass I (2007) Radiative and non-radiative charge recombination pathways in photosystem II studied by thermoluminescence and chlorophyll fluorescence in the cyanobacterium Synechocystis 6803. Biochim Biophys Acta Bioenergy 1767:233-243. https:// doi.org/10.1016/j.bbabio.2007.01.022

Eisenberg I, Caycedo-Soler F, Harris D, Yochelis S, Huelga SF, Plenio MB, Adir N, Keren N, Paltiel Y (2017) Regulating the energy flow in a cyanobacterial light-harvesting antenna complex. J Phys Chem B 121:1240-1247. https://doi.org/10.1021/acs.jpcb.6b105 90

Garcia-Pichel Ferran, Belnap Jayne, Susanne Neuer FS (2003) Estimates of global cyanobacterial biomass and its distribution. Arch Hydrobiol Suppl Algol Stud 109:213-227

Harel Y, Ohad I, Kaplan A (2004) Activation of photosynthesis and resistance to photoinhibition in cyanobacteria within biological desert crust. Plant Physiol 136:3070-3079. https://doi. org/10.1104/pp.104.047712

Heber U (2008) Photoprotection of green plants: a mechanism of ultra-fast thermal energy dissipation in desiccated lichens. Planta 228:641-650. https://doi.org/10.1007/s00425-008-0766-5

Heber U, Bilger W, Shuvalov VA (2006a) Thermal energy dissipation in reaction centres and in the antenna of photosystem II protects desiccated poikilohydric mosses against photo-oxidation. J Exp Bot 57:2993-3006. https://doi.org/10.1093/jxb/er1058

Heber U, Lange OL, Shuvalov VA (2006b) Conservation and dissipation of light energy as complementary processes: homoiohydric and poikilohydric autotrophs. J Exp Bot 57:1211-1223. https:// doi.org/10.1093/jxb/erj104

Heber U, Azarkovich M, Shuvalov V (2007) Activation of mechanisms of photoprotection by desiccation and by light: poikilohydric photoautotrophs. J Exp Bot 58:2745-2759. https://doi.org/10.1093/ jxb/erm139

Heber U, Soni V, Strasser RJ (2011) Photoprotection of reaction centers: thermal dissipation of absorbed light energy vs charge separation in lichens. Physiol Plant 142:65-78. https://doi.org/10.111 1/j.1399-3054.2010.01417.x

Ivanov AG, Sane P, Hurry V, Król M, Sveshnikov D, Huner NPA, Öquist G (2003) Low-temperature modulation of the redox properties of the acceptor side of photosystem II: photoprotection through reaction centre quenching of excess energy. Physiol Plant 119:376-383. https://doi.org/10.1034/j.1399-3054.2003.00225.x

Ivanov AG, Sane PV, Hurry V, Öquist G, Huner NPA (2008) Photosystem II reaction centre quenching: mechanisms and physiological role. Photosynth Res 98:565. https://doi.org/10.1007/s1112 0-008-9365-3

Jahns P, Holzwarth AR (2012) The role of the xanthophyll cycle and of lutein in photoprotection of photosystem II. Biochim Biophys Acta Bioenergy 1817:182-193. https://doi.org/10.1016/j.bbabi o.2011.04.012

Lakowicz JR (2006) Principles of fluorescence spectroscopy. Springer, New York

Miloslavina Y, Grouneva I, Lambrev PH, Lepetit B, Goss R, Wilhelm C, Holzwarth AR (2009) Ultrafast fluorescence study on the location and mechanism of non-photochemical quenching in diatoms. Biochim Biophys Acta Bioenergy 1787:1189-1197. https://doi. org/10.1016/j.bbabio.2009.05.012

Mullen KM, van Stokkum IHM (2007) TIMP: an R Package for modeling multi-way spectroscopic measurements. J Stat Softw 18:200-206. https://doi.org/10.1359/JBMR.0301229
Raanan H, Oren N, Treves H, Berkowicz SM, Hagemann M, Pade N, Keren N, Kaplan A (2016a) Simulated soil crust conditions in a chamber system provide new insights on cyanobacterial acclimation to desiccation. Environ Microbiol 18:414-426. https://doi. org/10.1111/1462-2920.12998

Raanan H, Oren N, Treves H, Keren N, Ohad I, Berkowicz SM, Hagemann M, Koch M, Shotland Y, Kaplan A (2016b) Towards clarifying what distinguishes cyanobacteria able to resurrect after desiccation from those that cannot: the photosynthetic aspect. Biochim Biophys Acta Bioenergy 1857:715-722. https://doi.org/10.1016/j. bbabio.2016.02.007

Ranjbar Choubeh R, Wientjes E, Struik PC, Kirilovsky D, van Amerongen $H$ (2018) State transitions in the cyanobacterium Synechococcus elongatus 7942 involve reversible quenching of the photosystem II core. Biochim Biophys Acta Bioenergy 1859:1059-1066. https://doi.org/10.1016/j.bbabio.2018.06.008

Sane PV, Ivanov AG, Sveshnikov D, Huner NPA, Öquist G (2002) A transient exchange of the photosystem II reaction center protein D1:1 with D1:2 during low temperature stress of Synechococcus sp. PCC 7942 in the Light lowers the redox potential of QB. J Biol Chem 277:32739-32745. https://doi.org/10.1074/jbc.M2004 44200

Slavov C, Reus M, Holzwarth AR (2013) Two different mechanisms cooperate in the desiccation-induced excited state quenching in Parmelia Lichen. J Phys Chem B 117:11326-11336. https://doi. org/10.1021/jp402881f

Snellenburg JJ, Laptenok SP, Seger R, Mullen KM, van Stokkum IHM (2012) Glotaran: a Java-based graphical user interface for the $\mathrm{R}$ package TIMP. J Stat Softw. https://doi.org/10.18637/jss.v049.i03

Tian L, Van Stokkum IHM, Koehorst RBM, Jongerius A, Kirilovsky D, Van Amerongen H (2011) Site, rate, and mechanism of photoprotective quenching in cyanobacteria. J Am Chem Soc 133:1830418311. https://doi.org/10.1021/ja206414m

Tian L, Gwizdala M, van Stokkum IHM, Koehorst RBM, Kirilovsky D, van Amerongen H (2012) Picosecond kinetics of light harvesting and photoprotective quenching in wild-type and mutant phycobilisomes isolated from the cyanobacterium Synechocystis PCC 6803. Biophys J 102:1692-1700. https://doi.org/10.1016/j. bpj.2012.03.008

Tian L, van Stokkum IHM, Koehorst RBM, van Amerongen H (2013) Light harvesting and blue-green light induced non-photochemical quenching in two different C-phycocyanin mutants of Synechocystis PCC 6803. J Phys Chem B 117:11000-11006. https://doi. org/10.1021/jp309570u

van Stokkum IHM, Larsen DS, van Grondelle R (2004) Global and target analysis of time-resolved spectra. Biochim Biophys Acta Bioenergy 1657:82-104. https://doi.org/10.1016/j.bbabi o.2004.04.011

van Stokkum IHM, van Oort B, van Mourik F, Gobets B, van Amerongen H (2008) (Sub)-Picosecond spectral evolution of fluorescence studied with a synchroscan streak-camera system and target analysis. In: Aartsma TJ, Matysik J (eds) Biophysical techniques in photosynthesis. Springer, Dordrecht, pp 223-240

Publisher's Note Springer Nature remains neutral with regard to jurisdictional claims in published maps and institutional affiliations. 